\title{
CHARACTERIZATION OF THE TEVATRON ELECTRON LENS MAGNETIC SYSTEM
}

\author{
K. Bishofberger; UCLA, Los Angeles, CA 90025, USA \\ V. Shiltsev; FNAL, Batavia, IL 60510, USA \\ A. Andriischin, A. Baluyev, S. Kozub, N. Krotov, V. Sytnik, A. Tikhov, L. Tkachenko; \\ Institute of High Energy Physics, Protvino, Russia \\ N. Solyak; Branch of Institute of Nuclear Physics, Protvino, Russia
}

\begin{abstract}
The Tevatron Electron Lens (TEL) installed in the Tevatron at FNAL bends a $10-\mathrm{keV}$ electron beam into and out of the antiproton path. The stringent requirements of the electron beam path demand extremely straight magnetic field lines over roughly two meters which then bend away to the gun and collector. A 6-Tesla superconducting solenoid and two normal-conducting solenoids, supplemented with several dipole and quadrupole correctors, were constructed at IHEP and subsequently assembled and tested at FNAL. Several testing methods, with an resolution of approximately $2 \mu \mathrm{rad}$, confirmed the field profile to match computer models and meet necessary constraints. Visual inspection of the beam position and size on screens confirmed the measurements. This paper analyzes these results and relates them to the system requirements.
\end{abstract}

\section{INTRODUCTION}

Other papers at this conference [1] actively discuss the physics of the Tevatron Electron Lens (TEL); in brief, a low-energy, high-current electron beam is produced, bent into the path of the antiproton beam in the Tevatron, allowed to transfer space-charge defocusing forces to the antiprotons (over about 2 meters), bent out again, and collected. This strong space-charge field will compensate beam-beam interaction effects, which severely limits the collider luminosity.

The electron beam energy is about $10 \mathrm{keV}$, and its horseshoe-type path is determined by a longitudinal field that the electron beam follows throughout its path. Since the electron beam must be collinear with the antiprotons, the magnetic field lines cannot deviate more than about $0.2 \mathrm{~mm}$ [2] during the interaction region and must connect to the cathode and collector on each side.

Confirmation that the magnets obey these requirements necessitated two field-measuring systems. The first was a 3-D Hall probe mounted on a $x y z$-translation table, which allowed accurate analysis of the bending magnetic field in the two elbows. The second used a magnetic needle on a small trolley that was pulled through the long solenoid, and achieved a resolution of $2 \mu \mathrm{rad}$.

\section{MEASUREMENT APPARATUS}

Both of the systems used LabVIEW programs to control stepper motors and read back sensor information. After the data was taken, information from both systems was overlapped to garner entire beam trajectories for several magnet configurations.

\subsection{3-D Hall Probe Apparatus}

An $x y z$-table held a rod where the probe was mounted. Using several rods of various length, the full range of motion along the longitudinal axis was $500 \mathrm{~mm}$; the table allowed $240 \mathrm{~mm}$ horizontal and $70 \mathrm{~mm}$ vertical travel transversely, and at every point the spatial resolution was $10 \mu \mathrm{m}$ in each direction. Figure 1 shows an illustration of the setup, which provided specific information of the magnetic field in the bending regions.

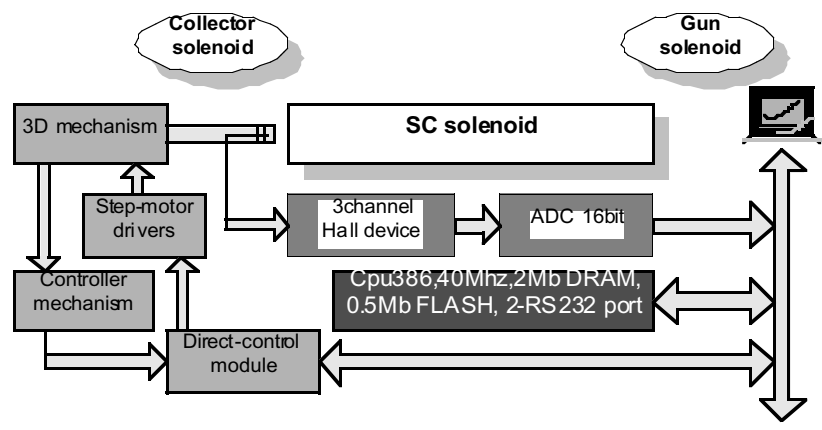

Figure 1: Block diagram of 3-D Hall probe measurement system.

In addition to measuring the total field produced by all of the magnets, the apparatus also inspected the fields in the gun and collector solenoids individually.

The LabVIEW programs successfully took data along a line, plane, or volume, and it also could follow a magnetic field line by evaluating field direction and directing the stage appropriately.

\subsection{Magnetic Needle Apparatus}

In order to get accurate field information along the entire length of the main solenoid, a small trolley was pulled along a long track through the length of the solenoid (Figure 2). The trolley held a freely rotating gimbal with a magnetic needle in the center. Attached to this needle was a mirror, and a laser was aimed at this mirror. The light reflecting off hit a position-sensitive detector 
(PSD), which reported back to LabVIEW the position where the laser beam hit.

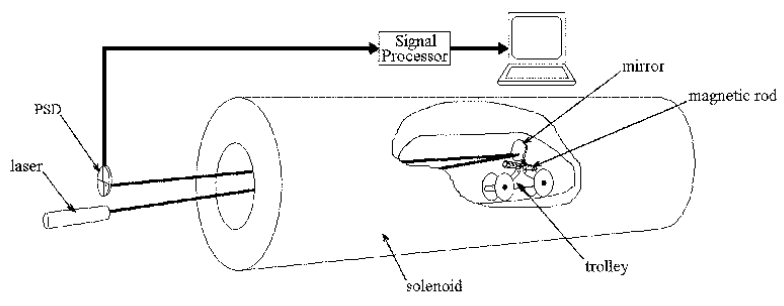

Figure 2: Schematic of magnetic-needle apparatus for field measurement.

Everything was aligned so that in the middle of the solenoid, the laser beam would reflect off of the mirror and hit the center of the PSD. As the trolley moved from end to end, small deviations in the magnetic field would translate into vertical and horizontal signals from the PSD, and geometry converted this information into the angle of the magnetic field along the solenoid, which could be integrated to trace the field lines.

The needle was about $5 \mathrm{~mm}$ diameter and $2 \mathrm{~cm}$ long, and therefore averaged the field over that region. However, moving the track transversely indicated an extremely uniform field, so the resolution was not an issue [3].

\section{CONVENTIONAL SOLENOIDS}

The conventional solenoid around the collector was designed with thicker flanges than the solenoid around the gun [4]. This increases the transfer function and reduces saturation effects in the collector solenoid, which is elucidated in Figure 3. The ellipticity $1-B_{y} / B_{x}$ of the solenoid field proved to be less than $\pm 0.2 \%$, the accuracy of the probe's sensitivity.

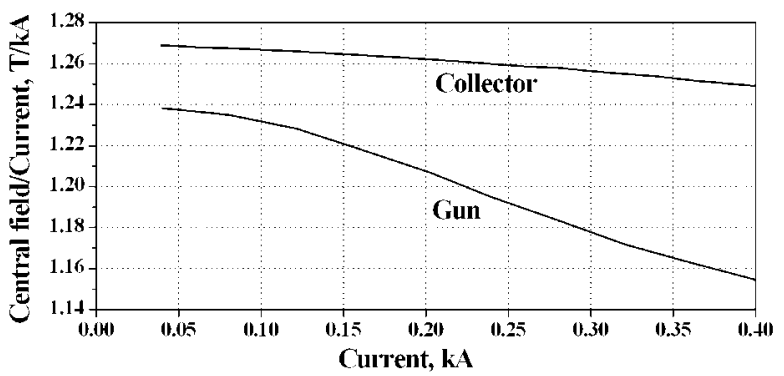

Figure 3: Transfer functions of the conventional solenoids.

Four 3.5-Amp corrector coils were built into each solenoid to provide dipole or quadrupole corrections to the field and, hence, the electron beam. The transfer function $B_{\max } / I$ for each dipole pair was measured to be about $19 \mathrm{G} / \mathrm{A}$, which, combined with the calculated magnetic length of $248 \mathrm{~mm}$, gives $16.5 \mathrm{kG}-\mathrm{mm}$ maximum integrated dipole field. The quadrupole transfer function was found to be $60 \mathrm{G} / \mathrm{mm} / \mathrm{A}$, which corresponds to
$52.1 \mathrm{kG} \cdot \mathrm{mm} / \mathrm{mm}$ maximum integrated quadrupole field or about $10 \%$ ellipticity.

Figure 4 illustrates the longitudinal field profile through each solenoid. The larger iron flange in the collector causes the higher skew in its profile.

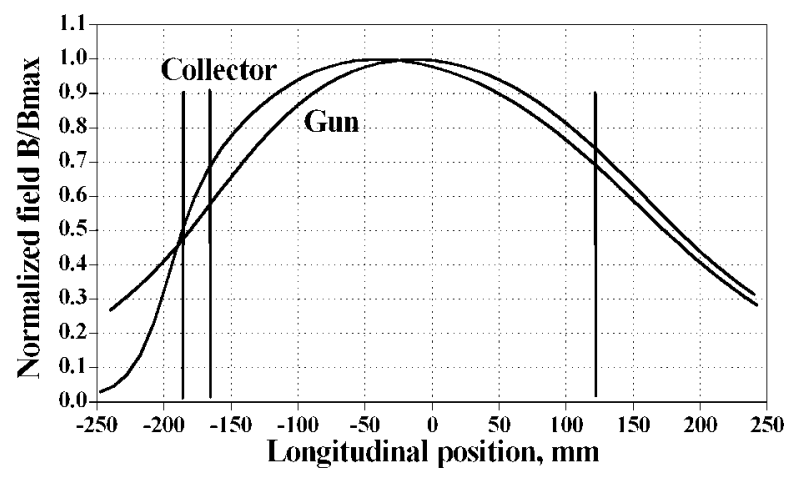

Figure 4: Field strength along the axes of the conventional solenoids.

\section{SUPERCONDUCTING SOLENOID}

The most stringent requirements on the magnetic system relate to the main superconducting solenoid. This solenoid must provide up to about six-Tesla longitudinal field and extremely small transverse field components. In addition, several corrector coils are needed to orient those field lines (and therefore the electron beam) along the antiproton trajectory.

During the initial testing, the solenoid quenched at 6.6 T; however, there was no subsequent quench through 6.7 T. The transfer function was measured to be $3.65 \mathrm{~T} / \mathrm{kA} \pm 1 \%$ throughout its range, nearly the calculated value of $3.66 \mathrm{~T} / \mathrm{kA}$. The normalized field along the longitudinal axis is shown in Figure 5.

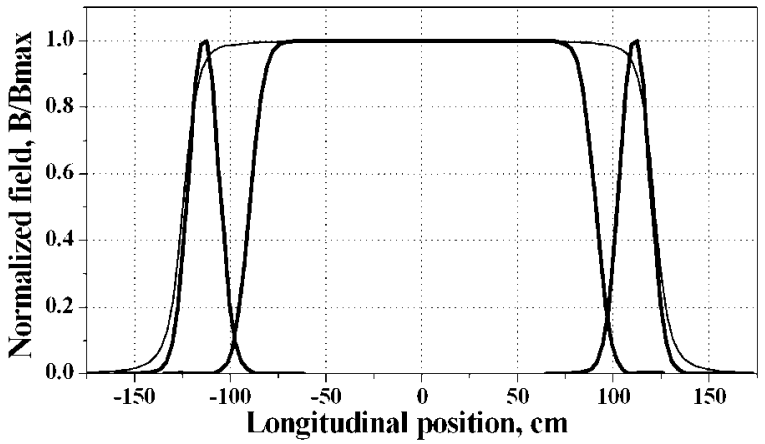

Figure 5: Main solenoid and corrector field distributions.

The corrector coils are divided into six dipoles: two short dipoles at each end of the solenoid and two more, longer dipoles extending along the central portion of the solenoid. The field strength for each segment is also shown in Figure 5. The short dipoles were normalized to $0.8 \mathrm{~T}$ and the longer dipoles to $0.2 \mathrm{~T}$.

The magnetic needle apparatus allowed observations of miniscule fieldline nonlinearities, which are displayed in 
Figure 6. At full power, the vertical deviations are very small (spanning about -25 to $25 \mu \mathrm{m}$ from the central axis with $15 \mu \mathrm{m} \mathrm{rms} \mathrm{deviation),} \mathrm{while} \mathrm{the} \mathrm{horizontal} \mathrm{devia-}$ tions have more spread (from -100 to $75 \mu \mathrm{m}$, or $50 \mu \mathrm{m}$ rms); however these values are still less than the required $\pm 0.2 \mathrm{~mm}$ tolerance. Figure 6 also depicts the field lines at half power, which are somewhat less straight.

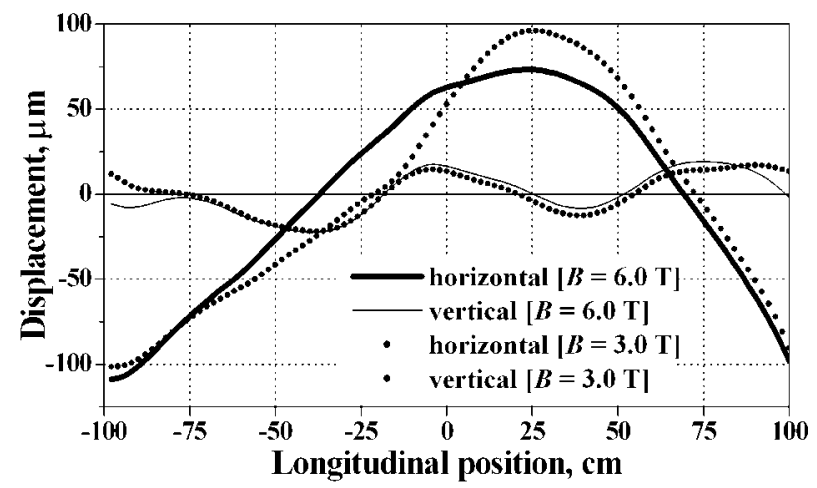

Figure 6: Deviations of field lines at full and half solenoid strength.

Figure 7 shows differences in field lines distributed horizontally from the central axis. Since the antiproton beam is not centered in the bore, uniformity of the field away from the axis is imperative, and the results indicate nearly parallel field lines.

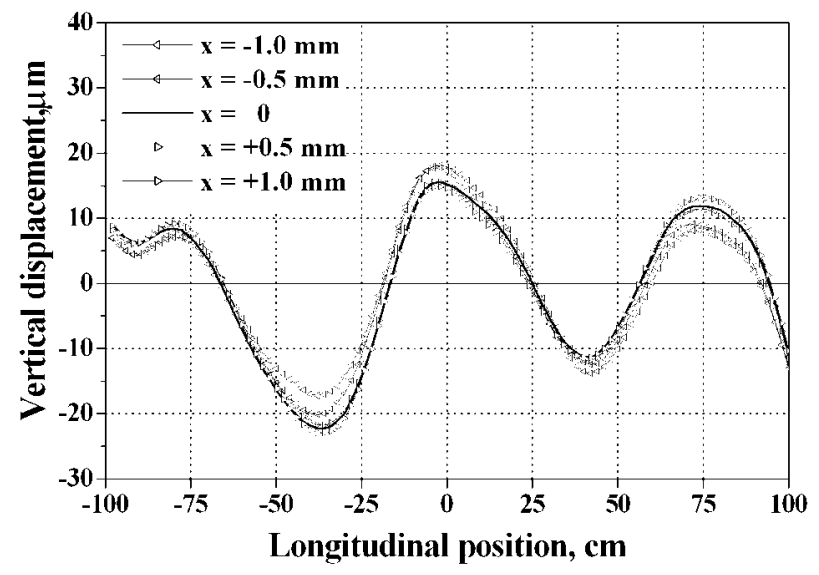

Figure 7: Magnetic field line deviations for a horizontally distributed array of field lines.

The complicated bending field lines between the superconducting solenoid and each of the conventional solenoids were mapped for a variety of configurations of solenoid and collector strengths. Figure 8 shows a few examples of how those field lines bend. The solid lines trace several initially displaced lines without use of correctors, and the dotted lines show how those lines changed with some field correction.

As a final check for the fieldline measurements, a screen was pulled through the superconducting solenoid while the electron beam was operating. The electron beam hitting the screen would glow and provide direct evidence of the

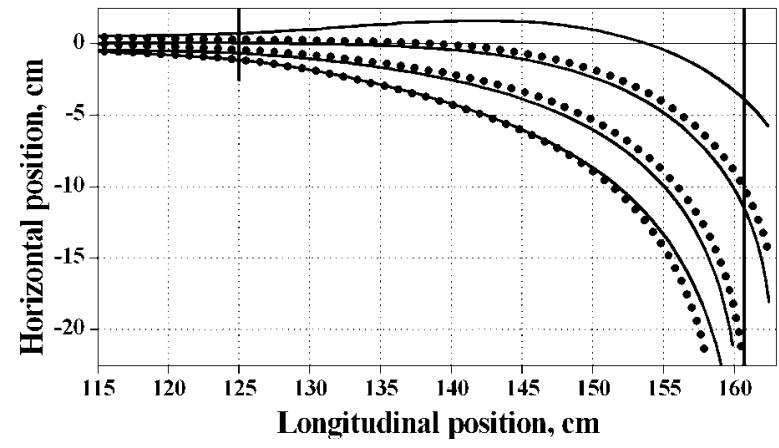

Figure 8: Field lines extending from main solenoid entrance (upper left) to collector solenoid (lower right).

transverse position of the lines along the length of the solenoid. Figure 9 shows both the horizontal and vertical position, but the screen could only be spied visually and qualitative, so the error bars on each point ranges from $0.2 \mathrm{~mm}$ to $0.5 \mathrm{~mm}$. Only a vague resemblance to the earlier data can be seen, but the large measurement errors excuse the deviations and disable quantitative analysis.

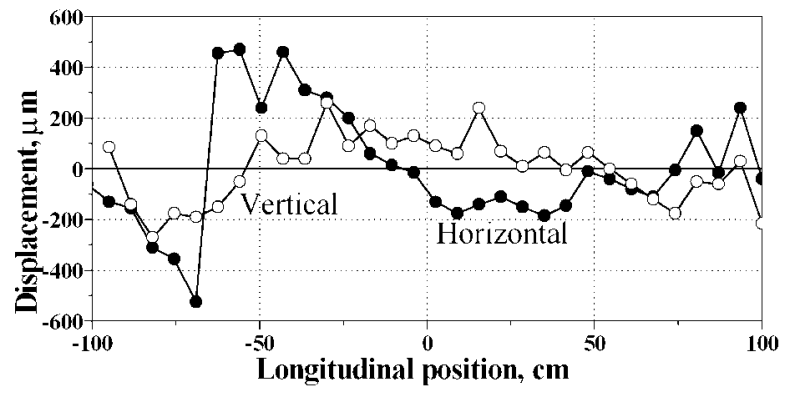

Figure 9: Visual inspection of the electron beam position.

\section{CONCLUSIONS}

A quench protection circuit was built to absorb as much. The magnetic characteristics of the Tevatron Electron Lens magnetic system have been measured to a high accuracy. All of these measured results are in good agreement with calculations, and they satisfy the requirements set by the physics of the TEL. Currently the project is operational in the Tevatron and has successfully affected its tune [1]. The magnetic system is stable and allows the rest of the project to move forward.

\section{REFERENCES}

[1] V. Shiltsev et al. "Beam-Beam Compensation in Tevatron: Status Report." This conference.

[2] D. Shatilov et al. "Analytical Study and Tracking Simulations of the Beam-Beam Compensation at Tevatron." This conference.

[3] C. Crawford et al. "Magnetic Field Alignment in the Beam-Beam Compensation Device." PAC-1999.

[4] A. Ageev et al. "Tevatron Electron Lens Magnetic System." This conference. 